\title{
EVALUATION OF SURFACTANT FUNCTION: IN VITRO AND IN VIVO TECHNIQUES IN EXPERIMENTAL RESPIROLOGY
}

\author{
Calkovska A., Mokra D.
}

Department of Physiology, Jessenius Faculty of Medicine, Comenius University, Martin, Slovak Republic

\begin{abstract}
Pulmonary surfactant is present as the thin film of surface active material in the terminal airspaces and conducting airways. The major function of the surfactant film is to reduce the surface tension at the alveolar surface. Deficiency or dysfunction of pulmonary surfactant cause severe respiratory diseases that make the study of pulmonary surfactant not only of physiological but also of clinical importance.

There are three main categories of methods for assessing the properties of pulmonary surfactant: in vitro, in situ and in vivo techniques. Pulsating bubble surfactometer (PBS) and captive bubble surfactometer (CBS) enables to study surfactant properties at spherical air-liquid interphase. Capillary surfactometer in contrast to alveolar models mimics the human terminal airways and evaluates surface properties required for airway patency. Each of above-mentioned methods enables to study exogenous surfactants, as well as surface activity of lavage fluids or tracheal aspirates.

Biophysical characteristics should be reflected by lung compliance and, as a consequence, by improved blood oxygenation. Various animal models have been developed to evaluate the efficacy of surfactant replacement therapy on preterm and term animals. In vivo models can be divided in those, primarily involving surfactant deficiency, such as premature animal model, and those with secondary surfactant dysfunction or inactivation, such as meconium or acid aspiration models.

This review is restricted to the in vivo and in vitro techniques handled by the authors in their research performed within the last years at both domestic and external laboratories.
\end{abstract}

Key words: Pulmonary surfactant, Surface activity, Surfactometer, Animal models

\section{INTRODUCTION}

Pulmonary surfactant is present as the thin film of surface active material in the terminal airspaces and conducting airways. The major function of the surfactant film is to reduce the surface tension at the alveolar surface. First, pulmonary surfactant reduces the energy required to inflate the lungs thereby increasing pulmonary compliance, second, by increasing elastic recoil, pulmonary surfactant reduces the likelihood of alveolar collapse during expiration. Deficiency or dysfunction of pulmonary surfactant causes severe respiratory disease. Exogenous surfactant replacement therapy, in which either synthetic or modified natural pulmonary surfactant is delivered into the patients lungs, has been established as a standard therapeutic intervention for patients with neonatal respiratory distress syndrome (RDS). Beside neonatal RDS, surfactant replacement therapy can also be helpful in other forms of lung disease in paediatric or adult patients, in which endogenous surfactant is inactivated by aspirated material or leakage of plasma proteins into the airspaces. Thus, with less or more success, exogenous pulmonary surfactant is also used in acute respiratory distress syndrome (ARDS), meconium aspiration syndrome (MAS), aspiration pneumonia and other forms of lung injury.

Methods for assessing the properties of pulmonary surfactant fall into three categories: in vitro, in situ and in vivo techniques. This review deals with the techniques used by the authors in the research done within the last years in laboratories of domestic and foreign institutions.

Address for correspondence:

Prof. Andrea Calkovska, MD, PhD, Department of Physiology, Jessenius Faculty of Medicine, Comenius University

Mala Hora 4, 03601 Martin, Slovakia, Phone/fax: +421 43 4131426, e-mail: calkovska@jfmed.uniba.sk 


\section{IN VITRO METHODS FOR EVALUATING PULMONARY SURFACTANT}

At least three physical properties of the surfactant system are essential for normal lung function, especially in the neonatal period. These are: 1) rapid adsorption from the hypophase, 2) low film compressibility with a drop in surface tension to very low values during surface compression and 3) effective replenishment of squeezed-out film constituents during surface expansion (1). Several systems have been used to determine the surface activity of surfactant materials. In this chapter, pulsating bubble surfactometer, captive bubble surfactometer and capillary surfactometer are discussed. Each of them enables to study exogenous natural or modified natural surfactants or newly developed synthetic surfactants, as well as surface activity of lavage fluid, tracheal or gastric aspirates after appropriate processing.

\section{Pulsating bubble surfactometry}

Pulsating bubble surfactometer was developed by Göran Enhörning and first published in 1977 (2). It enables to study surfactant properties at spherical air-liquid interphase. With this technique, approximately $20 \mu \mathrm{l}$ of test fluid is filled in a plastic sample chamber. An air bubble is connected to the ambient air by a chimney. A bubble of minimal radius $(\mathrm{r}=0.4 \mathrm{~mm})$ is created at $37{ }^{\circ} \mathrm{C}$ and it is maintained at minimal size for one to several minutes. Next, pulsation is started usually at a cycling rate of 20-40 rpm. The bubble radius then varies between $0.4-0.55 \mathrm{~mm}$ (corresponding to an area compression of $50 \%$ ). Pressure across the bubble wall is recorded during the $5^{\text {th }}$ cycle and after 1, 2 and 5 min of pulsation with a microprocessor and values for surface tension at maximum and minimum bubble size (Fig.1) are calculated according to the Laplace equation (e.g. 3). With this method, there are still problems with surface leaks. The method is not suitable to investigate the film stability at minimum surface tension in the non-pulsating mode. The pulsating bubble system is very convenient for examining the surface activity of samples under dynamic compression, as leakage is less of problem if relatively high cycling frequences are chosen (1).
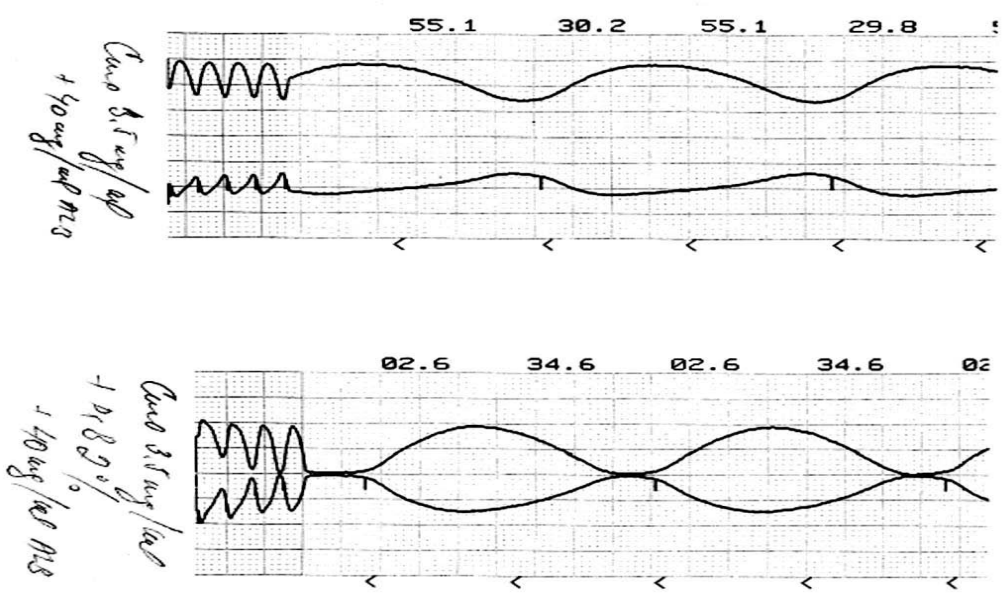

Fig. 1. Original recording from the pulsating bubble surfactometer reflecting different surface activity of the sample. Natural modified surfactant sensitive to inhibitor (up) and resistant to inhibitor (down). Differences in recordings are due to inability of inactivated surfactant to reach low surface tension at dynamic cycling. (Recordings were obtained during the study 3) 


\section{Captive bubble surfactometry}

Captive bubble surfactometer (CBS) was introduced in 1989 as an air-tight, leakage-proof alternative to pulsating bubble surfactometer (4). The test chamber is initially filled with $10 \%$ sucrose in saline. Two $\mu \mathrm{l}$ of surfactant are injected into the sample chamber and allowed to migrate by buoyance to the $1 \%$ agarose gel ceiling. An air bubble is then placed under the ceiling in contact with the surfactant preparation, and surface tension is measured from the time of bubble insertion. After $5 \mathrm{~min}$ of adsorption, the sample chamber is sealed, and the quasi-static cycling is initiated. Bubble volume is controlled by varying the pressure in the sample chamber. As bubble volume is reduced, the surface area is reduced and the surface tension of the surfactant film at the bubble surface falls. The bubble shape changes depending on surface tension, from more spherical to an oval shape. As the surface tension falls towards zero, a bubble shape assumes a thin disk. Minimum and maximum surface tension (Fig.2; 5), volume and area of compression needed to reach $\gamma \mathrm{min}$ of $5 \mathrm{mN} / \mathrm{m}$ (area \%) are calculated from the bubble height and diameter, from video images (6). The bubble is compressed stepwise until the surface tension $<5 \mathrm{mN} / \mathrm{m}$ is reached or to $50 \%$ area compression and thereafter expanded to the initial size. This manoeuvre is done repeatedly.

\section{Sample1}

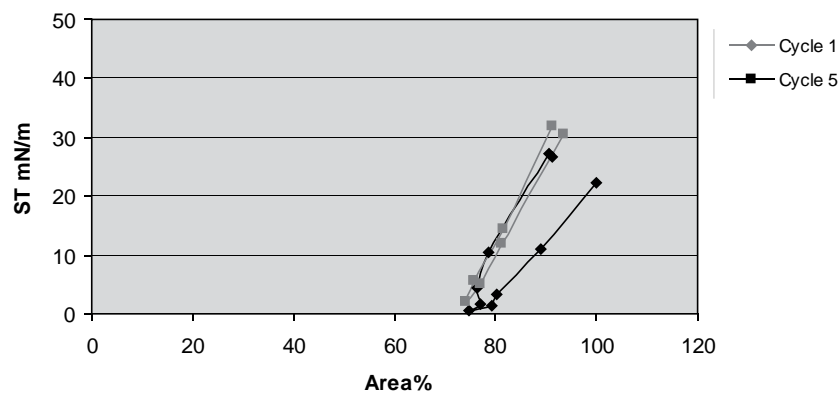

Sample 2

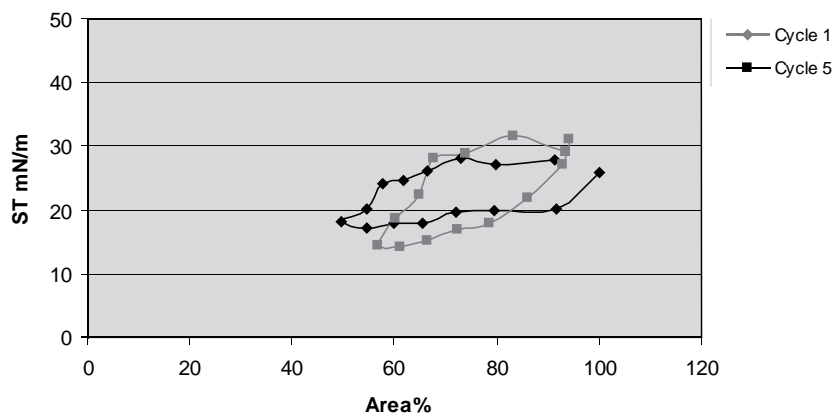

Fig. 2. Data obtained by measurement with captive bubble surfactometer. Upper figure represents active surfactant reaching minimum surface tension $\cong 0 \mathrm{mN} / \mathrm{m}$, lower figure shows inactivated sample inable to reach low minimum surface tension upon compression. (Recordings were obtained during the study 5)

Abbreviations: ST - surface tension (mN/m); Area \% - surface area compression needed to reach surface tension $\leq 5 \mathrm{mN} / \mathrm{m}$, Cycle 1 and Cycle 5 - first and last cycles of compression and expansion during measurement 


\section{Capillary surfactometry}

Capillary surfactometer is relatively new tool in the study of pulmonary surfactants (7). This model, in contrast to previous „alveolar“ models, mimics the human terminal airways. It evaluates surface properties required for airway patency. The sample at a volume of only $0.5 \mu \mathrm{l}$ is introduced into the narrow section of a glass capillary. At one end the capillary is connected to a bellows and a pressure transducer. When the bellows is slowly compressed, pressure is raised and recorded. The increasing pressure causes the sample to be extruded from the narrow section of the capillary.

As air gets through, pressure is abruptly lowered. If the sample contains well functioning pulmonary surfactant the sample liquid will not return to the narrow section. The steady airflow obtained by the continuous compression of the bellows will meet no resistance and the pressure recorded will be zero. On the other hand, at less active pulmonary surfactant, the sample liquid will return repeatedly. Ability to maintain the patency of the capillary (terminal conducting airways) is assessed by percentage of the total time (120 s) for which the capillary is open (Fig.3, 8).
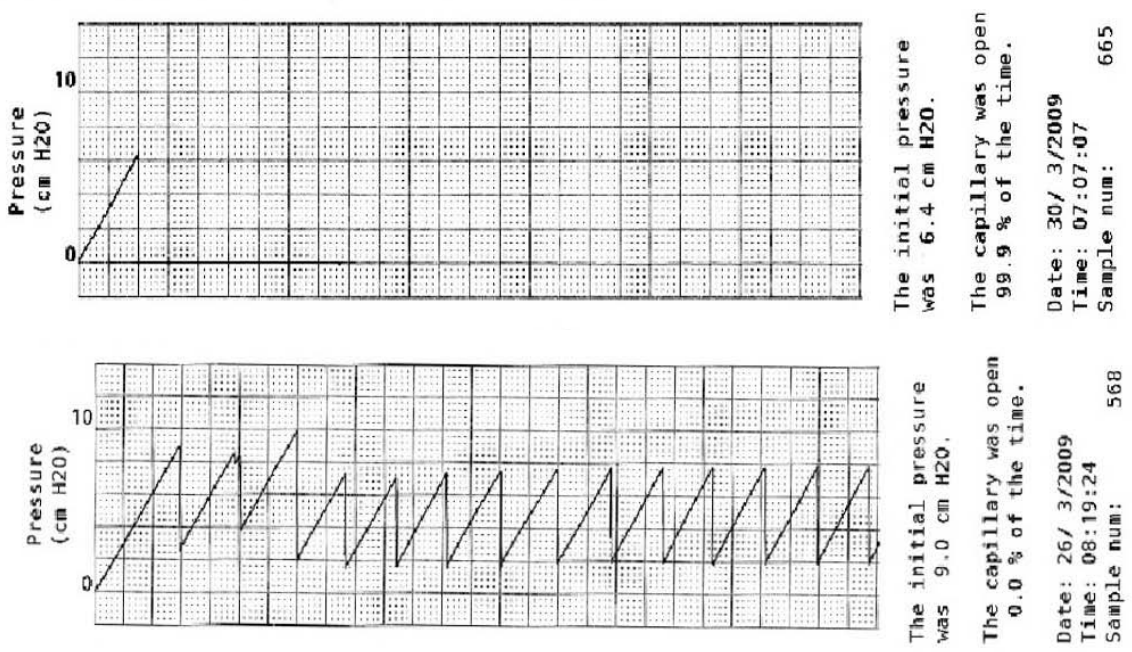

Fig. 3. Active surfactant does not return back to the narrow part of the capillary and thus, the capillary is open during the whole period of measurement (up); sample with inactivated surfactant requires repetitive generation of the pressure to open the capillary in effort to keep it patent (down) (from 8)

\section{ANIMAL MODELS FOR EVALUATION OF EXOGENOUS SURFACTANTS}

Biophysical characteristics of pulmonary surfactant should be reflected by lung compliance and, as a consequence, by improved blood oxygenation. However, the in vivo situation is more complicated since the physiological response is not only determined by the biophysical properties of the surfactant, but also by how the material is delivered to the terminal airspaces and how it is metabolized once deposited (9).

Various animal models have been developed to evaluate the efficacy of surfactant replacement therapy on preterm and term animals. In vivo models can be divided in those, primarily involving the surfactant deficiency, such as premature animal model, and those with secondary surfactant dysfunction or inactivation, such as meconium or acid aspiration models. 


\section{The immature newborn rabbit model}

Respiratory distress syndrom (RDS) is typically found in prematurely born infants. Key pathophysiological features include low lung compliance, low lung volumes and inadequate oxygenation. A relevant experimental model of neonatal RDS should be based on premature animals that do not have adequate amounts of surfactant in the fetal lung liquid and therefore fail to maintain proper lung aeration after birth. The animals should develop morphological evidence of hyaline membrane disease if allowed to breathe spontaneously or subjected to artificial ventilation. These criteria are met by several animal models, the most widely used prematurely delivered rabbits and lambs (10).

At 27 days of gestation (term=31 days), the fetal rabbit lung is nearly devoid of endogenous surfactant and the animals fail to develop adequate functional residual capacity. The animals are tracheotomized at birth and randomly allocated to different treatment groups. Up to ten animals are simultaneously kept in plethysmograph boxes at $37^{\circ} \mathrm{C}$ and ventilated in parallel with $100 \%$ oxygen. Depending on the protocol, different ventilatory strategies can be used. All animals are either ventilated with the same peak inspiratory pressure (PIP) with tidal volumes depending on lung function. As other modality, PIP and thus tidal volume are individualized for each animal. Beside the basic parameters of lung functions $\left(\mathrm{V}_{\mathrm{T}}\right.$, lung - thorax compliance), it is possible to evaluate post mortem lung gas volumes as an equivalent to functional residual capacity, and to do histological or morphometric studies (e.g. 5, 11). As an alternative lung lavage can be performed.

In this animal model it was shown for the first time that neonatal RDS can be prevented by surfactant replacement via airways (12) and these encouraging results stimulated the first clinical pilot studies of surfactant replacement therapy for neonatal RDS (13). Moreover, this model can be a base for e.g. neonatal meconium aspiration model (14) or neonatal pneumonia model $(15,16)$. This model has also been utilized extensively to test exogenous surfactants. In those studies the level of positive end-expiratory pressure (PEEP) should be carefully standardized. The use of PEEP during mechanical ventilation influences several parameters of surfactant function, including dynamic lung compliance and lung permeability, and these effects may differ for different surfactants.

One of the advantages of the preterm rabbit model is the size of the preterm rabbit lung which ensures optimal surfactant distribution through the lung with a relatively simple instillation technique. Moreover, the pregnant rabbit doe usually carries multiple fetuses - ten or more in each litter that makes possibile to do the measurements relatively fast by using a large number of animals simultaneously $(10,17)$. On the other hand, the small size of these experimental animals (average body weight about $30 \mathrm{~g}$ at a gestational age of 27 days) precludes more invasive pathological studies (e.g. intravascular administration).

\section{Surfactant-depletion model by lung lavage}

Surfactant-depletion experimental model was developed in adult guinea-pigs (18). It is a rather clean model of surfactant depletion, involving no other initial insult to the pulmonary parenchyma. In this model, severe respiratory insufficiency is defined as a fall in $\mathrm{PaO}_{2}$ below $60 \mathrm{mmHg}$ during appropriate artificial ventilation with 100\% oxygen. This method involves connecting the animal to a mechanical ventilator and subsequently lavaging the lung with saline to remove the endogenous surfactant. Because the lavage procedure can evoke the secretion of intracellular surfactant, it should be repeated several times at regular intervals. Blood gases, parameters of lung mechanics, and histologic and electron microscopic findings indicated that the lavage procedure induces a condition similar to the adult respiratory distress syndrome. Already the first lavage results in significant reduction of lung-thorax compliance and repeated lavage in a pertubation of lung functions persisting for at least $8 \mathrm{~h}$. The most relevant outcome of surfactant function in these studies will be blood oxygenation (17). The original protocol has been modified slightly by other investigators. The lung lavage models are useful for a variety of experimental purposes, including testing of surfactant preparations, evaluation of pharmacological agents stimulating synthesis and 
secretion of alveolar phospholipids and studies on the significance of various ventilator settings. In our studies, we used lung-lavaged adult rabbits to test the delivery method of exogenous surfactant and the effect of ventilation strategy after treatment (19).

\section{Model of meconium aspiration}

Surfactant function may be disturbed in several forms of neonatal lung disease other than RDS. For example, surfactant may become inactivated by aspirated meconium or by leaking plasma proteins in inflammatory conditions characterized by increased alveolar permeability. The first experimental demonstration of meconium aspiration was reported by Gooding et al. (20) who injected human meconium into the trachea of newborn dogs. The presence of aspirated meconium in peripheral airspaces was verified by radiological and histological examination. Surfactant inhibition by meconium in experimental animals was first demonstrated by Chen et al. (21), who studied the quasi-static pressure-volume characteristic of the lungs after airway instillation of 10\% human meconium. Since then only six years were needed to publish results on first severely ill babies with meconium aspiration syndrome (MAS) treated with exogenous surfactant (22).

Surfactant dysfunction by meconium was proved in various animal models that have been used to evaluate the efficacy of different ventilatory strategies, surfactant replacement and other pharmacological therapy. This model is extensively used by our group (e.g. 23). Today, due to technical difficulty with newborn animals, the adult ones are favoured. Usually, human meconium from healthy full-term newborn infants is used. It is pooled, lyophylized and stored frozen. Prior administration, meconium is diluted by saline to appropriate concentration and injected intratracheally into anesthetized and paralyzed animals into right and left position. Animals are then artificially ventilated. Respiratory failure is defined as a $\geq 30 \%$ decrease in dynamic lung-thorax compliance and $\mathrm{PaO}_{2} \leq 10 \mathrm{kPa}$ at $\mathrm{FiO}_{2}$ 1.0. Pathophysiological and morphological consequences in this model of newborn MAS are due to combination of airway obstruction, chemical pneumonitis and surfactant inactivation $(14,24)$.

The model imitates to a great extent the aspiration of meconium in a newborn, however, the interpretation of results must be done carefully. Animals are usually healthy before meconium instillation that does not correspond to hypoxic human newborn and moreover, dynamics of spontaneous meconium aspiration differs from that after syringe instillation. In spite of these concerns, experimental model of meconium aspiration is a valuable tool in evaluation of many aspects of clinical MAS therapy (for review, see 25).

\section{CONCLUDING REMARKS}

In vitro modelling provides results that can be obtained faster than in animal studies. It is also less expensive and enables larger flexibility of experimental conditions. The problem is that not all in vivo studies confirm the previous in vitro data. In vivo the situation is more complex since the physiological response is not only determined by the biophysical properties of the surfactant, but also by many factors arising from the living organism.

Moreover, stimulating results obtained in animal experiments are not always reproducible in clinical trials. Firstly, most animal studies have reported on respiratory failure caused by direct injury whereas clinical acute lung injury is caused often by indirect insult. Secondly, in animals, surfactant is usually administered in optimal timing whereas in patients this treatment tends to be late. Finally, most of the animal studies are terminated within several hours and thus, they cannot evaluate long-term effects of surfactant therapy. In spite of all these concerns in vitro and in vivo modelling has a constant position in the surfactant research.

\section{REFERENCES}

1. Robertson B, Schurch S. Assessment of surfactant function. In: Uhlig S, Taylor AE, editors. Methods in pulmonary research. Basel-Boston-Berlin: Birkhäuser Verlag; 1998. p. 349 - 83.

2. Enhorning G. Pulsating bubble technique for evaluating pulmonary surfactant. J Appl Physiol 1977; 43: $198-203$. 
3. Calkovska A, Some M, Linderholm B, Johansson J, Curstedt T, Robertson B. Biophysical and physiological properties of porcine surfactant enriched with polymyxin B. Biol Neonate 2005; 88: 101-8.

4. Schürch S, Bachofen H, Goerke J, Possmayer F. A captive bubble method reproduces the in situ behavior of lung surfactant monolayers. J Appl Physiol 1989; 67: 2389 - 96.

5. Calkovska A, Linderholm B, Haegerstrand - Björkman M, Curstedt T. Fibrinogen counteracts inactivation of lung surfactant by surface area cycling. Neonatology 2010; 97(4): 398-9.

6. Schoel WM, Schurch S, Goerke J. The captive bubble method for the evaluation of pulmonary surfactant: surface tension, area and volume calculations. Biochim Biophys Acta 1994; 1200 (3): 281 - 90.

7. Lema G, Enhorning G. Surface properties after a simulated PLA2 hydrolysis of pulmonary surfactant's main component, DPPC. Biochim Biophys Acta 1997; 1345(1): 86-92.

8. Mokra D, Tribulova E, Saffova I, Erneyova J, Calkovska A. Changes in surface activity of surfactant induced by in vitro exposure to meconium assessed by capillary surfactometer. Acta Med Mart 2010; 10(3): 13-18,

9. Lewis JF, Veldhuizen RA. Factors influencing efficacy of exogenous surfactant in acute lung injury. Biol Neonate 1995; 67: 48-60.

10. Robertson B. Animal models of neonatal surfactant dysfunction. In: Robertson B, van Golde LMG, Batenburg JJ, editors. Pulmonary surfactant. From molecular biology to clinical practice. Amsterdam, London, New York, Tokyo: Elsevier, 1992. p. 459 - 84.

11. Calkovska A, Some M, Linderholm B, Curstedt T, Robertson B. Therapeutic effects of exogenous surfactant enriched with dextran in newborn rabbits with respiratory failure induced by airway instillation of albumin. Pulm Pharmacol Ther 2008; 21(2): 393-400.

12. Enhörning G, Robertson B. Lung expansion in the premature rabbit fetus after tracheal deposition of surfactant. Pediatrics 1972; 50: 58-66.

13. Fujiwara T, Maeta H, Chida S, Morita T, Watabe Y, Abe T. Artificial surfactant therapy in hyalinemembrane disease. Lancet 1980; 1: 55-9.

14. Sun B, Curstedt T, Song GW, Robertson B. Surfactant improves lung function and morphology in newborn rabbits with meconium aspiration. Biol Neonate 1993; 63: 96-104.

15. Herting E, Jarstrand C, Rasool O, Curstedt T, Sun B, Robertson B. Experimental neonatal group B streptococcal pneumonia: effect of a modified porcine surfactant on bacterial proliferation in ventilated near-term rabbits. Pediatr Res 1994; 36(6): 784-91.

16. Stichtenoth G, Linderholm B, Björkman MH, Walter G, Curstedt T, Herting E. Prophylactic intratracheal polymyxin B/surfactant prevents bacterial growth in neonatal E. coli pneumonia of rabbits. Pediatr Res 2009; [Epub ahead of print]

17. Zuo YY, Veldhuizen RAW, Neumann AW, Petersen NO, Possmayer F. Current perspectives in pulmonary surfactant - inhibition, enhacement and evaluation. Biochim Biophys Acta 2008; 1778: 1947 - 77.

18. Lachman B, Robertson B, Vogel J. In vivo lung lavage as an experimental model of the respiratory distress syndrome. Acta Anesthesiol Scand 1980; 24(3): 231-6.

19. Calkovska A, Mokra D, Javorka K, Petraskova M, Adamicova K. Exogenous surfactant administration by asymmetric high-frequency jet ventilation in experimental respiratory distress syndrome. Croat Med $\mathrm{J}$ 2005; 46(2): 209-17.

20. Gooding CA, Gregory GA, Taber P, Wright R. An experimental model for the study of meconium aspiration of the newborn. Radiology 1971; 100: 137- 40.

21. Chen CT, Toung TJK, Rogers MC. Effect of intra-alveolar meconium on pulmonary surface tension properties. Crit Care Med 1985; 13: 233-6.

22. Auten RL, Notter RH, Kendig JW, Davis JM, Shapiro DL. Surfactant treatment of full-term newborns with respiratory failure. Pediatrics 1991; 87: 101-7.

23. Mokra D, Calkovska A, Drgova A, Javorka M, Javorka K. Treatment of experimental meconium aspiration syndrome with surfactant lung lavage and conventional vs.asymmetric high-frequency jet ventilation. Pediatr Pulmonol 2004; 38(4): 285-91.

24. Moses D, Holm BA, Spitale P, Liu M, Enhorning G. Inhibition of pulmonary surfactant function by meconium. J Obstet Gynecol 1991; 164: 477-81.

25. Mokra D, Mokry J. Meconium aspiration syndrome: from pathomechanisms to treatment. New York: Nova Biomedical Books; 2010.

\section{Acknowledgement}

This work was supported by project "Center of Experimental and Clinical Respirology" No. 26220120004, cofinanced from EU sources.

Received: January, 12, 2011

Accepted: February, 7, 2011 